\title{
Monitoring and Controlling Services for Electrical Distribution Systems Based on the IEC 61850 Standard*
}

\author{
Rodrigo Prat, Gustavo Rodriguez, Fernando Magnago \\ Facultad de Ingeniería, Universidad Nacional de Río Cuarto, Río Cuarto, Argentina \\ E-mail: fmagnago@ing.unrc.edu.ar \\ Received April 18, 2011; revised April 30, 2011; accepted May 10, 2011
}

\begin{abstract}
International standards are being developed to promote rapid configuration and integration into the utility automation system. In order to take advantage of modern technology to provide new benefits to users of substation automation, the International Electrotechnical Commission (IEC) has developed and released a new global standard for substation automation, named IEC 61850. This aim of this paper is to provide a basic technical overview of substation automation and the standard IEC 61850. It will discuss the benefits of each major aspect of this standard and it will describe how these concepts can be used in the development and implementation of tools related to Substation Automation. The application of communication standards and protocols to facilitate data exchange within the substation environment is presented. In addition, the model is implemented using a small distribution system that plans to utilize new communication technology following the IEC 61850 standard. Finally it proposes a set of laboratories to be used as the core of an undergraduate course. Results illustrate that the communication approach as proposed by IEC 61850 fulfills the real-time requirements for control and protection of distribution system.
\end{abstract}

Keywords: Substation Automation, IEC 61850

\section{Introduction}

The main function of electric power systems (EPS) is to produce, transmit and deliver energy to the users. Within this field there are important issues that need to be considered which cause many undesirable effects such as service interruptions and outages produced by equipment failures, natural storms or accidents. Although to mitigate these effects have always been important, this importance has increased recently due to the evolution of the demand that requires uninterrupted and high quality of electric power.

Therefore, the challenge for the EPS is to provide a quick response to the undesirable effects, maintaining reliability and competitive prices. Within this scenario, having high quality real time information becomes a very important concern. One way to improve this is through fully integrated, automated and remotely supervised systems that require minimal human intervention [1].

Consequently, automation of power distribution systems has increasingly been adopted by power utilities worldwide in recent years as part of their efforts to pro-

*This work was supported by CONICET (Argentina) vide a more reliable supply to its customers and to enhance operational efficiency. Thus, the principal objective of electric system automation is to respond rapidly to real time events with pre-specified actions in order to maintain uninterrupted power service [2].

Although the systems already use automation, nowadays an additional subject is becoming important: the growing share of disperse and renewable generation. Therefore the traditional assumption that the electricity flow direction goes unidirectional from higher to lower voltage levels does not stand any longer. The increasing penetration of distributed generation into the system results on bidirectional power flow and, as a consequence, the architecture and technology used for system automation need to address this new system structure [3].

Hence, electric system automation infrastructure needs to increase the level of intelligence and integration of information and communication technologies (ICT). Current efforts related to the innovation and the development of this topic is known as Smart Grid [4]. The Smart Grid vision of electrical system, remarks the importance and benefits of communications to deliver real time information and enables the near instantaneous bal- 
ance supply and demand at device level [5].

Under these circumstances, standardization plays a crucial role in building up an intelligent power system grid.

Many researchers and several international organizations are currently developing the required communication technologies and the international communication standards for electric system automation [6].

In addition, as the understanding of these topics becomes very important for power engineers, the incorporation of practical experiences on this matter in the undergraduate curricula is fundamental [7], together with the design of new laboratories to expose students to real life situations within this area.

This paper discusses the state of the art of communication standards and protocols to facilitate data exchange within the substation environment and proposes a set of educational laboratories to expose undergraduate students to this technology. First, it remarks the advantages of substation automation; second, it describes the evolution of substation communication, gives an introduction to Intelligent Electronic Devices (IED) and their impact on the substation communication architecture, and then, provides a basic technical overview of IEC 61850, where its main features are highlighted. Besides, the paper discusses the benefits of each major aspect of the standard, and describes how they can be used in the development and implementation of systems for different substation level applications. Finally, an implementation of monitoring and controlling services for electrical distribution systems employing the IEC 61850 standard is presented. This implementation is designed as a set of laboratories to be implemented into the undergraduate curricula.

\section{Substation Automation Systems}

The principal function of the Substation Automation System (SAS) is to provide SCADA RTU (Remote Terminal Unit) functionality. The initial function was to concentrate data from the substation into one or more messages for transmitting them to the master station [8]. Nowadays, the Substation Automation System (SAS) also comprises protection as well as control, monitoring, and communication functions that furnishes all functions required for the safe and reliable operation of the substation. It allows operating substations based on accurate information provided in a timely manner to the decision making application and devices. Moreover, it incorporates several advantages to the management of the distribution systems. These advantages are presented in different areas: operation, customer services, and financial performance.
From all the advantages the following can be highlighted:

- Enhanced operational efficiency.

- Improved communication.

- Better-quality customer services.

- Improved financial performance.

- Maximized incentives.

Although the concept of SAS is not new, it becomes popular now due to the current needs and the evolution of communication technologies. Initially the SAS architecture made use of a master-slave architecture in a star topology and used vendor specific proprietary protocols. Among the limitations, it can be remarked that the user was bound to one vendor, there was no direct communication between slaves and the master communication represented a bottleneck.

In 2002 Ethernet became available for speeds up to 10 Gbps which provides standardized high speed communication. Furthermore, the introduction of distributed functionalities replaced the master-slave structure and the development of enhanced and comprehensive security, made SAS architecture to improve drastically [1]. In addition, electronic devices technology improved as well. The development of Intelligent Electronic Devices (IED) typically consists of one or more microprocessors and communication ports with the ability to transmit data and execute control commands, which makes further development for SAS architectures possible.

\section{Intelligent Electronic Devices}

Advances in digital electronics and communication technology have enabled improvements on RTUs and become Internet-ready Intelligent Electronic Devices. The main functions of a typical IED include protection, control, monitoring, metering and communications. Integrating these IEDs into electric systems can offer several benefits, among others, remote access to the relay configuration ports, diagnostic event information, and video for security or equipment status assessment. Nowadays, in SAS, the accurate functionality of a system depends on the integrity and the interoperability of IEDs from different manufacturers. The key objectives for designing the substation automation architecture are the interoperability between IEDs, the satisfaction of communication performance, and the extensibility of the architecture.

As we move into the digital age, literally thousands of analog and digital data points are available in a single IED, being communication bandwidth no longer a limiting factor.

The evolution of the technology developments within this area are explained next. 


\section{Evolution of Substation Communication}

Until 1985 there were no standards, few vendors provide basic devices for substation, interoperability was not considered and all systems were proprietary.

These first devices did not use the same physical interfaces or protocols, even the same vendors developed devices with communication protocols that were incompatible.

The network architecture was employed a master-slave network topology and communication between substations was not possible. The communication methods were based on RS232, RS485, phone line, radio, and high voltage transmission lines. The speed was up to 1200 bits per second, and the used protocols were Modbus, SEL, WISP, or Conitel 2020.

Initially, the communication systems applied for SAS were proprietary; therefore interconnection between devices from different vendors, which were few, was very difficult.

From 1985 to 1990 more vendors provide devices for Substation Automation, some protocol converters allow basic interoperability among different devices and the use of microprocessor decentralized architecture make redundancy and multiple masters possible. New advances on technology permit the communication speed to increase up to 19200 bps. The most popular protocols were DNP3 and the first version of IEC 870.

From 1990 the need for standardization and the evolution of the technology change this scenario. Developing of interoperability from physical (connectors, voltage levels) to communication protocols becomes an important issue.

Local Area Network (LAN) was introduced in substation applications, different substations were able to be communicated using Wide Area Networks (WAN). The technology applied was Frame Relay, ATM, and Ethernet, and communication speed increases over 1 Mbps. Protocols like Transport Control Protocol/Internet Protocol (TCP/IP), Hypertext Transfer Protocol (HTTP) and File Transfer Protocol (FTP) started to be used within the stations. Several protocols were adapted to work on TCP/IP like Modbus, DNP3, IEC 60870 and initial work on standardization started.

The development of standards started in 1994 when EPRI started a project named UCA (Utility Communication Architecture). Based on the requirements, the compilation of different existing international standards in data networks and computer communication was done $[9,10]$.

In 1996 the IEC Technical Committee 57 started a similar standardization project named IEC 61850 [5], then, in 1997 both groups joined to define a single stan- dardization under the name IEC 61850. As a result of these agreements, in 2004, a new version of the standard was released [11]. Finally in 2009, the standard incorporated the Distributed Energy Resources (DER) [12]. The main features of this standard are described in the following section.

\section{Features of the Standard IEC 61850}

International standards are being developed to promote rapid configuration and integration into the utility automation system [19]. The IEC 61850 presents the international standard for communication networks and system in substation automation

The approach of the standard IEC 61850 is founded on the separation of the object model with its data and services from the communication. This allows both to follow the state-of-the-art communication technology (stack) and to safeguard the investments in functions and databases (object model). Therefore, the standard is future-proof as far as possible. Among its main features, the following can be highlighted:

- Interoperability by various manufacturers IEDs as an integrated system;

- High data transfer among IEDs considering peer to peer communication model instead of master-slave communication model in recent protocols;

- Data definition based on advanced object-oriented model which contains whole data specifications instead of single-oriented model with each data definition by numeric addresses;

- Supporting functionality of devices to provide better communication;

- Communications extend ability and data integrity;

- Providing integrated communication system;

- Providing robust management of substation automation system.

Three important aspects are considered in the norm: a comprehensive data model (Object model), the communication service to access and exchange data, and the Substation Configuration Language (SCL).

\subsection{Object Models}

The data modeling of the IEDs are defined following an object oriented modeling. One of the main objectives of the norm is to establish the interoperability among devices from different vendors, therefore the communication and interaction is based on an abstract communication architecture that provides a hierarchic class set with associated services which are protocol independent. The norm defines a service model, named Abstract Communication Service Interface (ACSI), which is used for data 
and service definition.

The IED ACSI models in a hierarchic order are:

- Server: represents what can be seen from a device, all other models are part of the server;

- Logical Device (LD): contains the information produced by a group of functions of specific applications. Functions are defined as logical nodes;

- Logical Node (LN): contains the information produced by a specific function;

- Data: provides the tools to define the type of information included in a LN.

These basic ACSI information models are illustrated

in Figure 1.

Based on these models, a client application under IEC 61850 is able to know the complete structure of the IED, or the portion that is allowed to use. This information can be obtained in a recursive manner and built on a hierarchical structure using the associated services, among which the most important are GetServerDirectory, GetLogicalDeviceDirectory, GetLogicalNodeDirectory, Get AllDataValues, GetDataValue and SetDataValue.

These services are mapped into specific application and communication protocols are defined in the norm by a Specific Communication Service Mapping (SCSM).

\subsection{Communication}

\subsubsection{Communication Levels}

From the communication point of view, SAS can be considered divided into three different levels:

- Process level: the lowest level which includes sensors, current and voltage transformers, circuit breakers, and any devices needed for the monitor and operation of the substation;

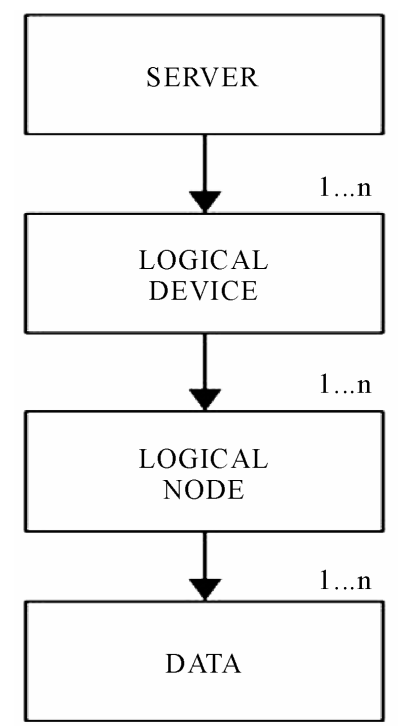

Figure 1. ACSI information models hierarchical structure.
- Bay level: includes control and protection devices, can also include functions related with the operation of other bays;

- Substation level: it is the highest level; it includes local consol central units (gateways) that are connected to the control center.

\subsubsection{Protocol Stacks}

The norm defines the use of different protocols depending on the transmitted message type. The sample values, GOOSE messages and the GSSE are directly mapped onto the data layers; therefore, these types of messages can not be directed to another network. Simple Network Time Protocol is used to synchronize messages. The Abstract Communication Service Interface is mapped onto the Manufacturing Message Specification layer [9]. These different layers are shown in Figure 2.

\subsection{Substation Configuration Language (SCL)}

This language is a formal way to describe how individual devices are configured; that is, what data and services they are supposed to be supporting.

This language is an XML based language, following a W3C XML Schema. Four file types which describe the automation requirements and devices are defined. These files are: IED Capability Description file, extension ICD, describes the IED capacity. Configured IED Description, extension CID, describes the IED configuration for a specific project. System Specification Description, extension SSD, describes the substation and logical nodes required. Substation Configuration Description, extension SCD, describes the communication, all IEDs used, and the substation. The norm establishes the files structures which contains a header, a substation description, the IEDs configuration, the communication system, and the data type. These data are mandatory or optional depending on the file type as illustrated in Table 1.

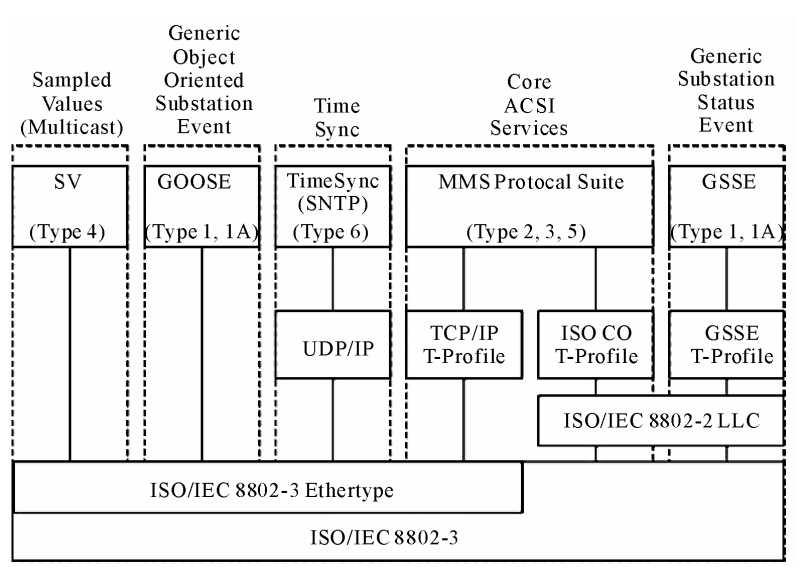

Figure 2. IEC 61850 communication protocols profile. 
Table 1. SCL file sections.

\begin{tabular}{ccccc}
\hline Structure & SSD & ICD & SCD & CID \\
\hline Header & yes & yes & yes & yes \\
Substation & yes & Optional & yes & $\begin{array}{c}\text { Optional } \\
\text { IED }\end{array}$ \\
Optional & $\begin{array}{c}\text { Yes, opt. } \\
\text { values }\end{array}$ & Multiple & $\begin{array}{c}\text { Yes, } \\
\text { including } \\
\text { values }\end{array}$ \\
Communication & Optional & $\begin{array}{c}\text { One } \\
\text { instance }\end{array}$ & yes & $\begin{array}{c}\text { One } \\
\text { instance } \\
\text { Data type }\end{array}$ \\
If needed & If needed & Multiple & If needed \\
\hline
\end{tabular}

\section{Implementation}

A prototype of intelligent distributed control services applied to the current electrical distribution system of the National University of Rio Cuarto (UNRC) has been implemented.

The first objective of the implementation is to study basic concepts of the standard IEC 61850 architecture and attempts to describe how these concepts can be used in the development of substation automation systems. The second one is to develop a set of laboratories in order to make power engineering students understand these concepts in a real scenario. Therefore, the implementation is organized as a sequence of didactic laboratories.

The current electrical distribution system of the UNRC is used to make these practical laboratories. The distribution system consists of six $13.2 \mathrm{kV}$ feeders as described by the one line diagram of Figure 3. It is connected to the local utility at the MAIN substation which provides electricity to different regions of the university.

Derived from the diagram of Figure 3, a set of laboratories is designed to make students understand how to build the substation automation system (SAS) schematic following the steps recommended by the standard. These laboratories are explained in the next subsections.

\subsection{Laboratory 1: SAS Schematic Diagram Design}

In order to simplify the problem, only MAIN substation is considered, since the same analysis can be extended to the other substations. The purpose of the Laboratory is to build the schematic diagram derived from the IEC 61850 standard. As a result of work done in this laboratory, all needed logical nodes (LN) that represent the different SAS functionalities are selected. Figure 4 shows the final SAS scheme for the substation. For this substation only over current relays were included as protection devices. The used LNs are:
- IHMI: operator interface;

- TVTR: voltage transformer;

- TCTR: current transformer;

- XCBR: circuit breaker to be tripped;

- CSWI: switch controller;

- PTOC: AC time over current protection;

- MMXU: measuring.

Figure 4 describes the different LNs proposed for the SAS, TVTR, TCTR, and XCBR are the LNs at process level, PTOC. MMXU, and CSWI at bay level and the local consol representation IHMI is the LN at the substation level.

\subsection{Laboratory 2: Substation Configuration Language Files Generation}

On the basis the one line diagram shown in Figure $\mathbf{3}$ and the schematic diagram illustrated in Figure $\mathbf{4}$ as a result of laboratory 1 , the objective of this laboratory is to generate the SCL files that are needed by the system. By working in this laboratory students become familiar with the file generation required by the standard.

In order to generate these files in a timely manner, an evaluation SCL manager program selected by the students is used [13].

First, the SSD (System Specification Description) file which represents the substation one line diagram with the required LNs is built. Figure 5 illustrates the generated diagram in accordance with the SSD format, where also the IED and the communication section are added. From the IEDs, the ICD (IED Capability Description) files are generated; these files are provided by the IEDs vendors and represent their capabilities.

Second, the necessary IEDs are placed in order to fulfill the requirements imposed in the SSD file. Next, the IED network parameters are configured. After that, the file SCD (Substation Configuration Description) based on the SSD file and the links between the imposed LNs and the IED's LNs are generated. Finally, the CID (Configured IED Description) files are generated based on the IED configuration, these files are the ones that will be uploaded into the IEDs.

\subsection{Laboratory 3: Equipment Selection}

The aim of this laboratory is to select the appropriate equipment based on the design done in previous tasks. Although the requirement was to explore different alternatives, the protection devices at the selected substation were already present, and then the objective was modified to study if the current equipment were appropriate for the design done.

Based on the selected equipment, a client server ap- 


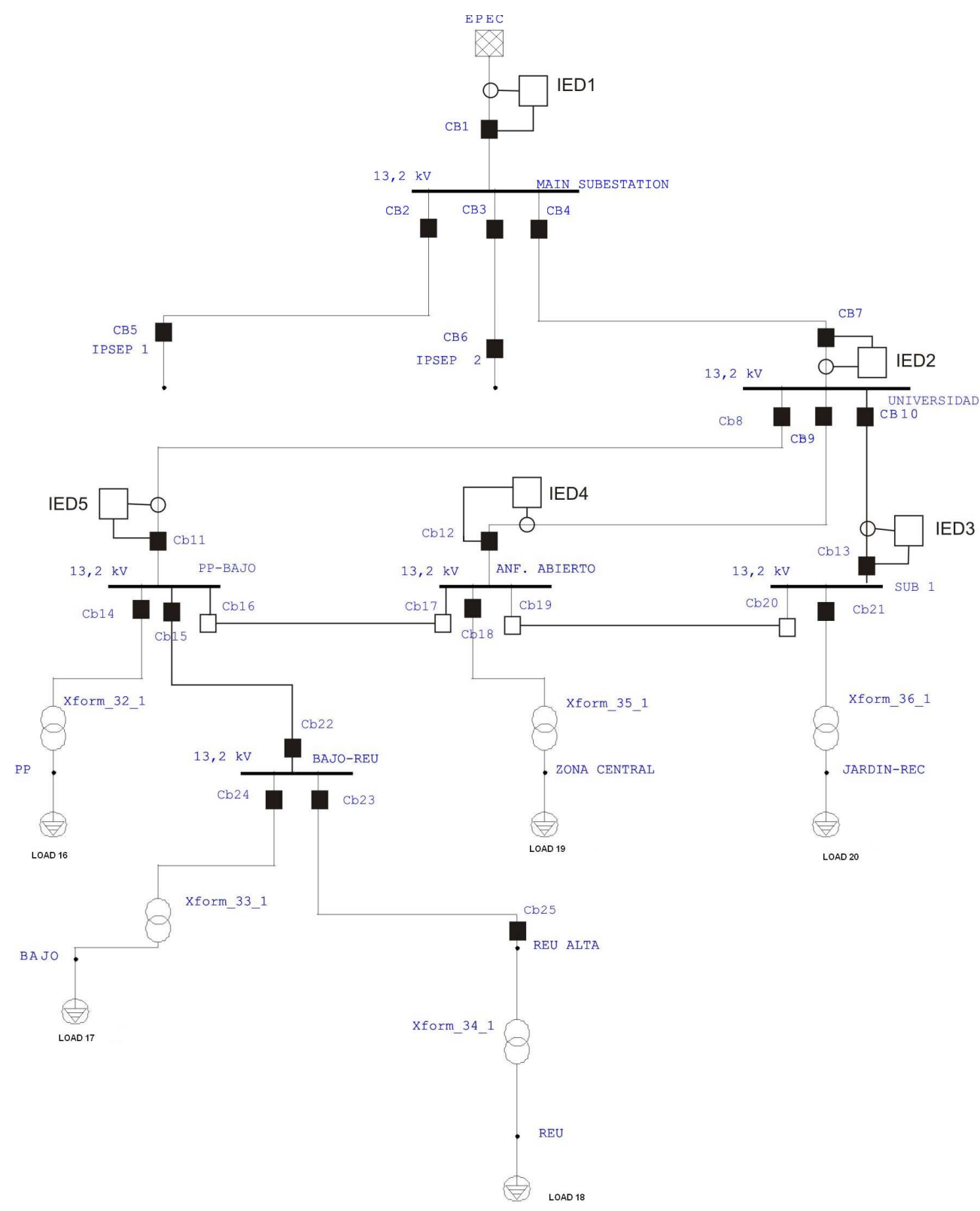

Figure 3. One line diagram.

plication is designed and developed to emulate a generic IED.

\subsection{Laboratory 4: IED Prototype Design}

The system specification description file (SSD) has been developed in Laboratory 2. This file describes the substation schematic and the logical node requirements which represent the specific functions that will be used for the system automation. These functions, per substation, are:

- XCBR: monitoring of circuit breaker;

- TCTR: monitoring of current transformer;
- CSWI: control of circuit breaker;

- PIOC: over current protection.

Figure 6 illustrates one of the substations mapped onto logical nodes derived from the standard IEC 61850 architecture. These LNs are included into three different Logical Devices (LD): one at the bay level, the second at the process level, and the last one at the substation level.

At process level, the IED has one logical device (LD1) defined; this logical device has two different logical nodes, the circuit breaker logical node (LD1.LN1) XCBR and the current transformer logical node (LD1.LN2) TCTR. They represent the device specific data and provide services as defined in the standard. 


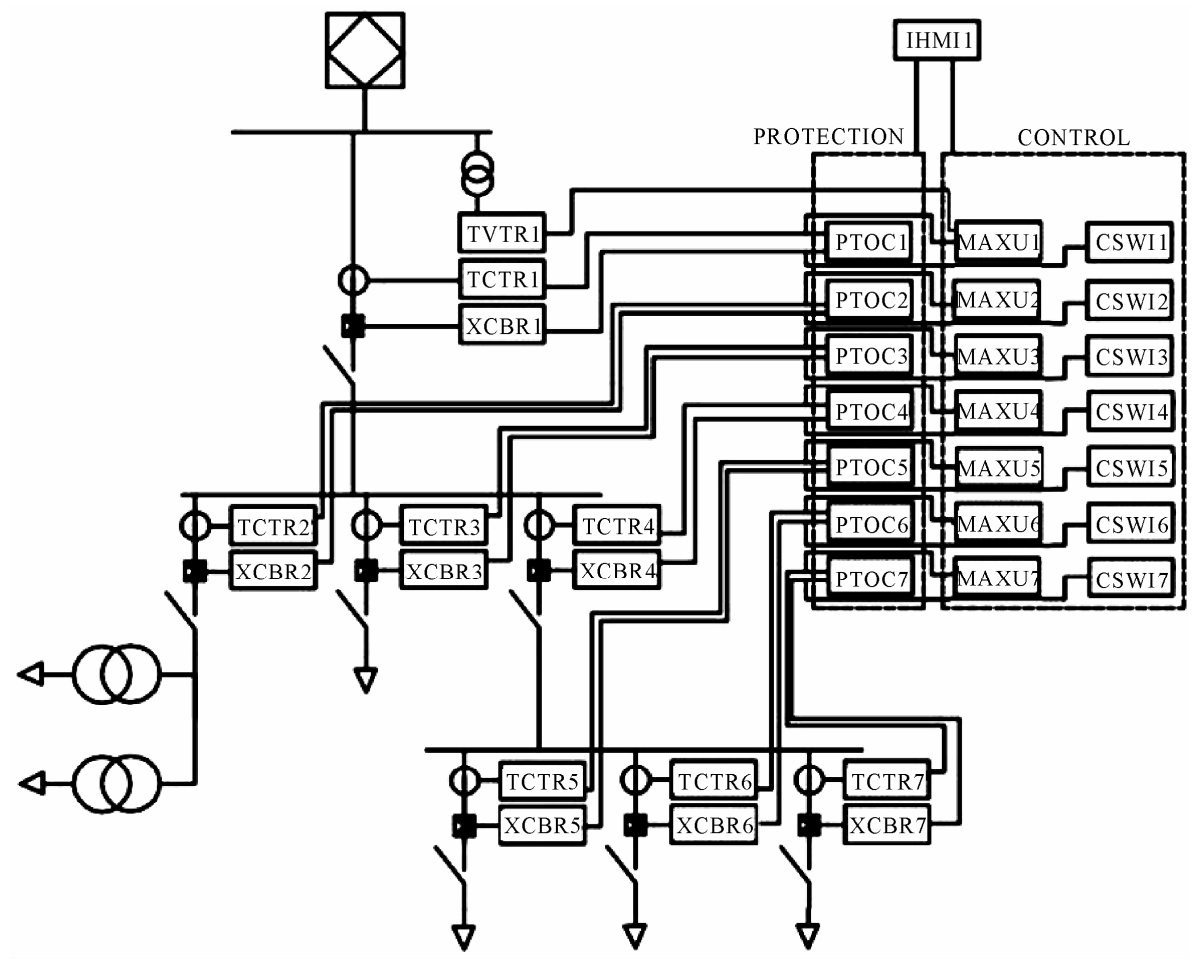

Figure 4. Reduced SAS scheme.

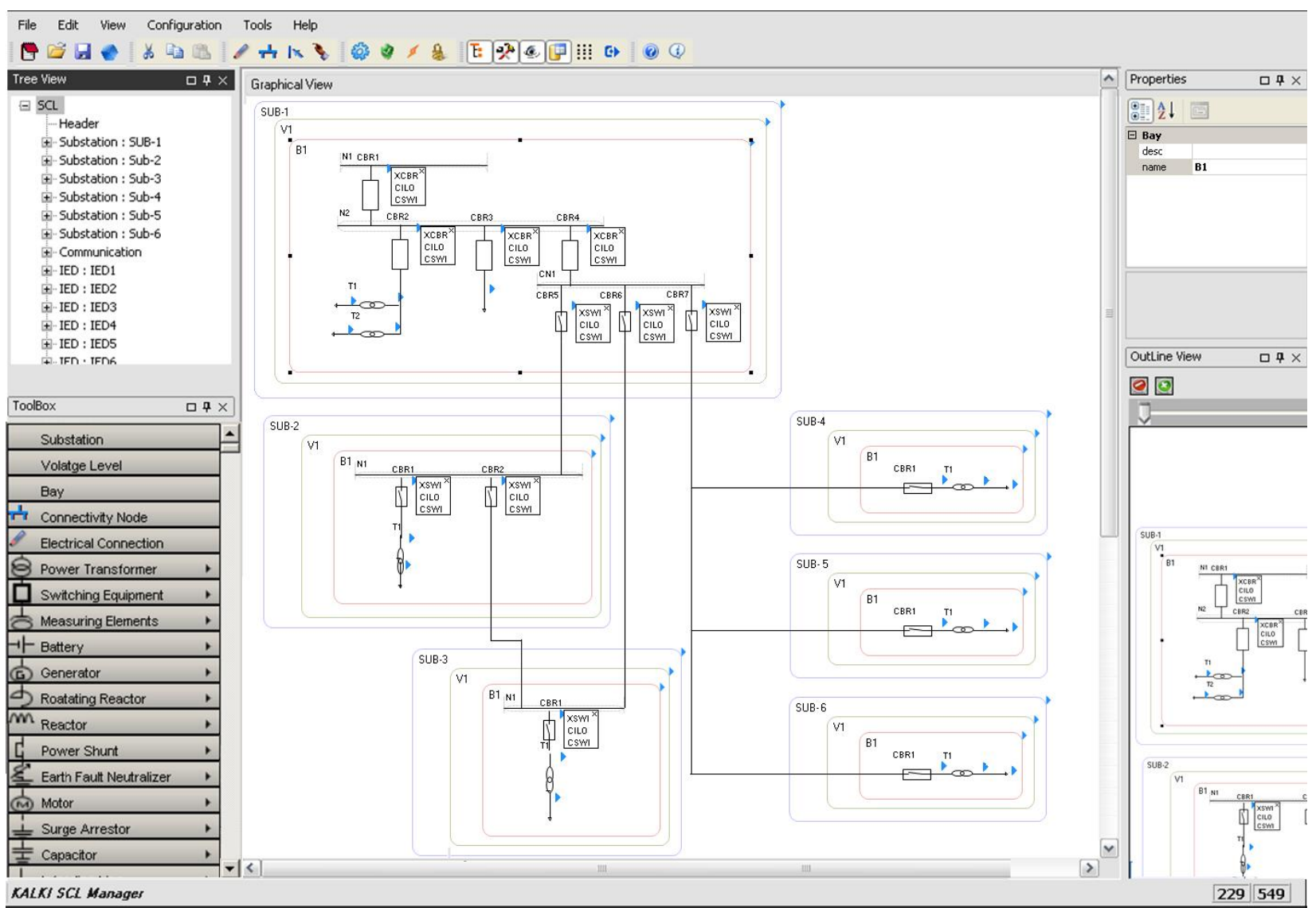

Figure 5. SSD diagram. 


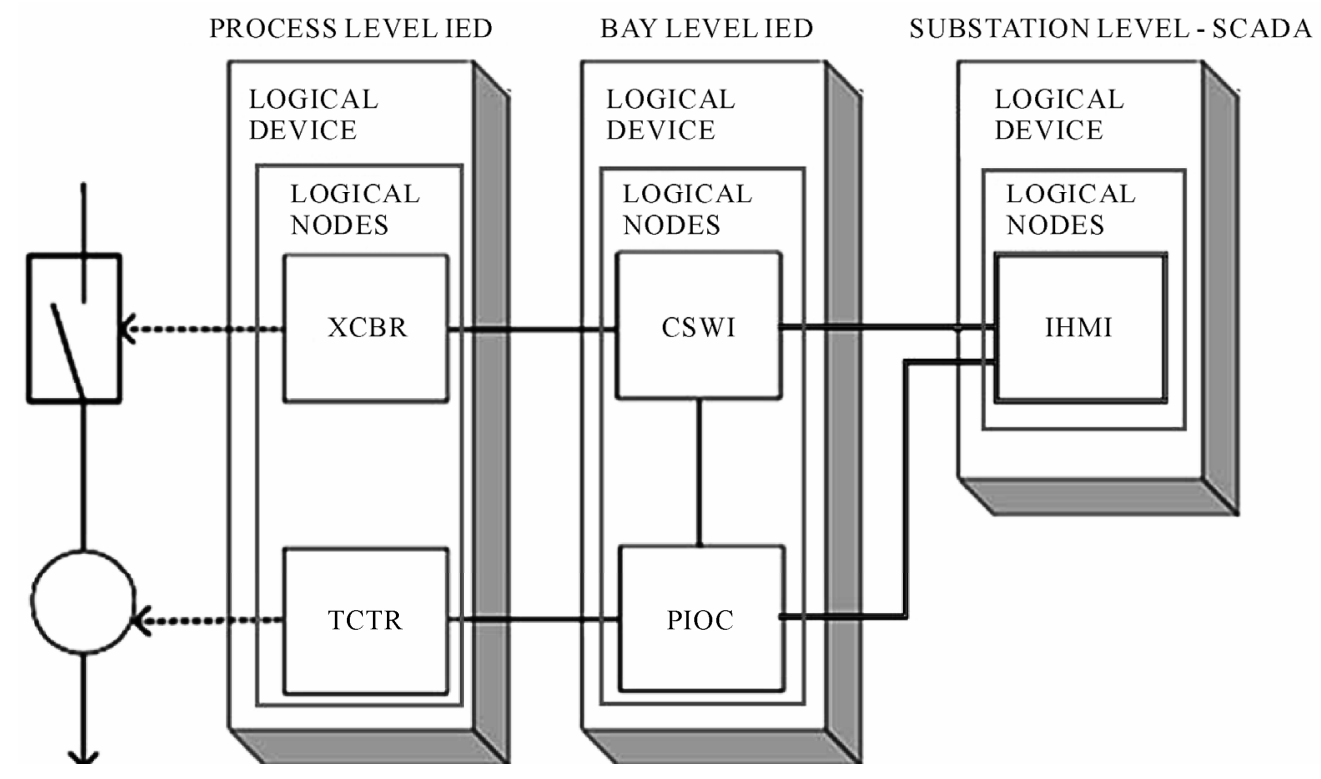

Figure 6. Protection and measurement virtualization.

XCBR provides the circuit breaker status and TCTR senses the current. Figure 7 shows the virtual device of the circuit breaker logical node and the corresponding data as well as the data attributes based on the standard definitions.

At the bay level, another logical device (LD2) is defined within its IED, this logical device has also two different logical nodes, the circuit breaker control logical node (LD2.LN1) CSWI and the over current relay logical node (LD2.LN2) PIOC. Based on the information provided by the logical node at process level, PIOC has the function of detecting the fault, and then CSWI has the function of isolating the fault. Finally, the local SCADA at the substation level has one LN, the IHMI, which can visualize and control the data of the logical nodes present at the bay level (CSWI and PIOC).

Each one of the LNs defines the design of the IEDs; at the process level; at the bay level and at the substation level. Based on this design, two simple IEDs were materialized as a server - client application subsystem for one of the substation. The server is implemented at the bay level, while the client application is implemented at the substation level.

\subsection{Laboratory 5: IED Prototype Implementation}

Based on the design, in this assignment, a server-client application is developed in order to emulate two basic IEDs. The main objective of this implementation is to study the basic concepts of the standard, to visualize the structure of an IED and to become familiar with the communication and information interchange among dif- ferent IEDs.

Figure 8 shows the diagram indicating the server-client structure for the IEDs and the SCADA. The SCADA access as a client of the bay level server, while the IED located at the bay level access as a client of the IED server located at the process level. In order to simplify the analysis, only the server-client application that evolves the bay level IED and the local SCADA is described. The implementation is developed in $\mathrm{C}++$ under Microsoft Windows XP, where the information objects and the communication modules are developed. The server application is explained below.

\subsubsection{Server Application}

From the standard, different modules have been implemented; the main focus of the developed modules has the purpose of studying the information and communication stages of the standard. These modules are explained next:

- Initialization module: known as Substation Configuration Language (SCL), this module extracts configuration parameters from a configuration file “.icd”|cite \{SCL\}. For example, extracts network parameters such as IP address, and gateway, and system parameters such as LNs and LDs. Then it sends the parameters to different modules in order to have a proper system initialization;

- Information module: this module implements the classes related to the complete model specified by the file ".icd" from the parameters given by the initialization module SCL. This model consists of a set of classes named server, logic device, logic node, data, and data attribute. This model has a hierarchic and nested structure, where the server is the higher object. 


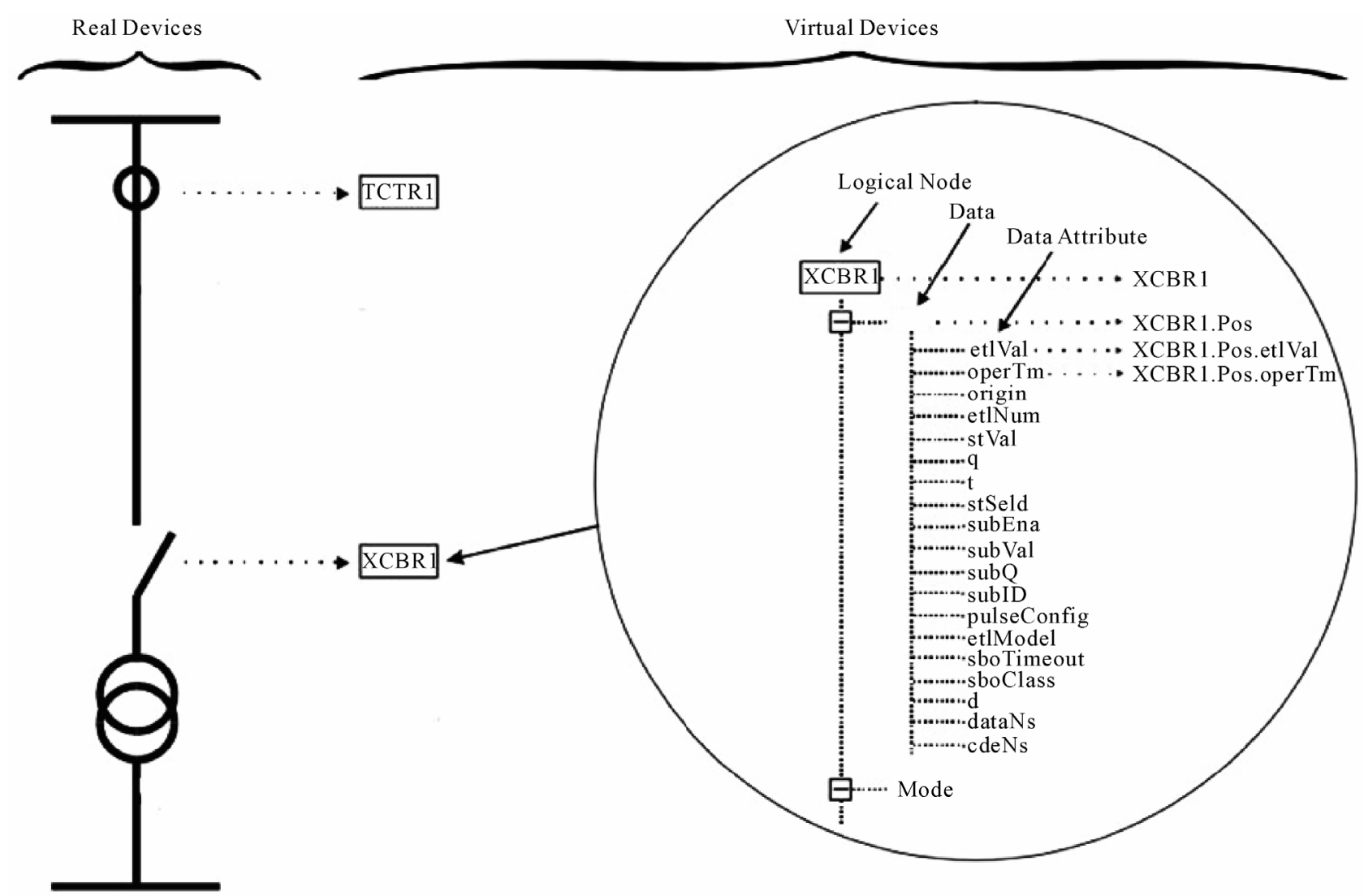

Figure 7. Circuit breaker real and virtual description.

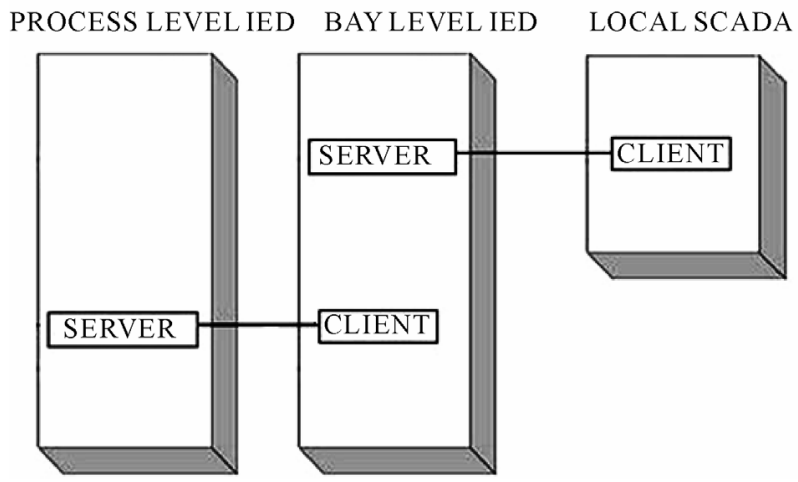

Figure 8. Server-client UML diagram.

An object of the server class contains objects of logic device class, objects of the logic node class have objects of data class and finally objects of the data class have objects of data attribute class., and the last one have the data value. Figure 9 describes the methods and attributes implemented within the different classes that conforms the basic information model. As an illustration of the developed methods, Figure 10 shows one of the most basic methods;

- Communication module: has as the main task the communication with the clients. For this specific im- plementation, the communication system was implemented using sockets TCP for information interchange within the network. The server waits for petition information from the client using a TCP/IP network model. The syntax used for the client server messages was developed under the IEC 61850 standard.

\subsubsection{Client Application}

To implement client application, a simple application that allows testing the server performance has been developed. Different methods have been developed to access the server services. Communication protocols that make use of TCP sockets have been implemented. The client sends a query to the server, which processes the petition and answers accordingly.

Figure 11 and Figure 12 show the server and console screen shots respectively which illustrate the steps during the communication when the client located at the bay level asks for information regarding the logical nodes at the process level.

\section{Conclusions}

In this paper, a model of a small distribution system that 


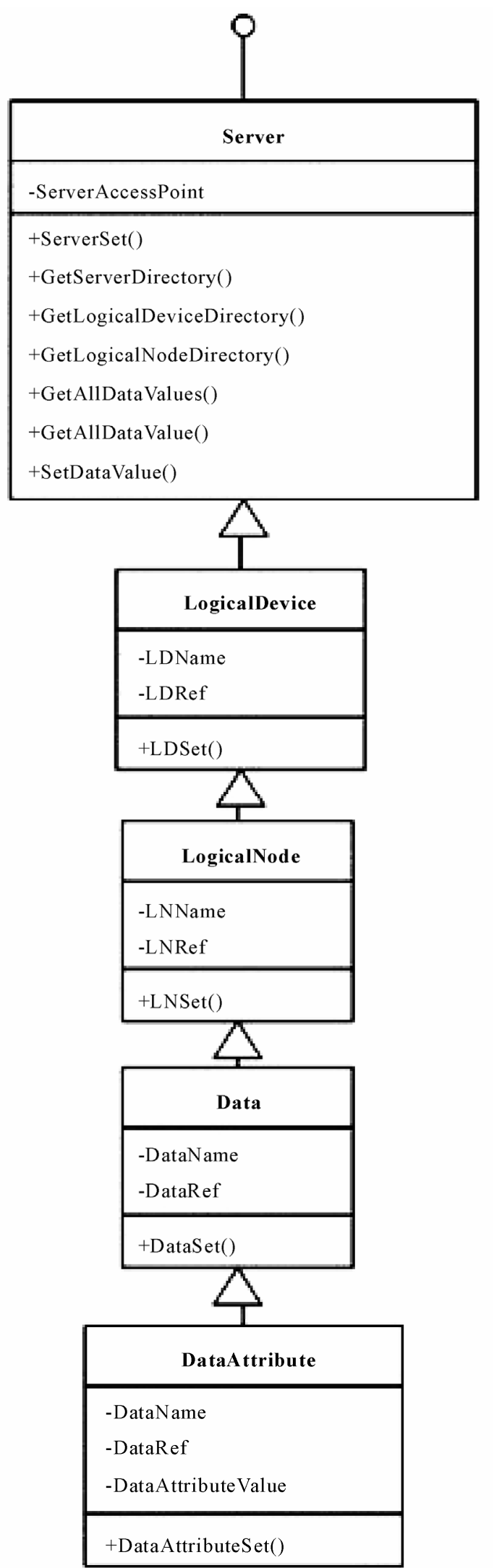

Figure 9. Information model UML diagram.

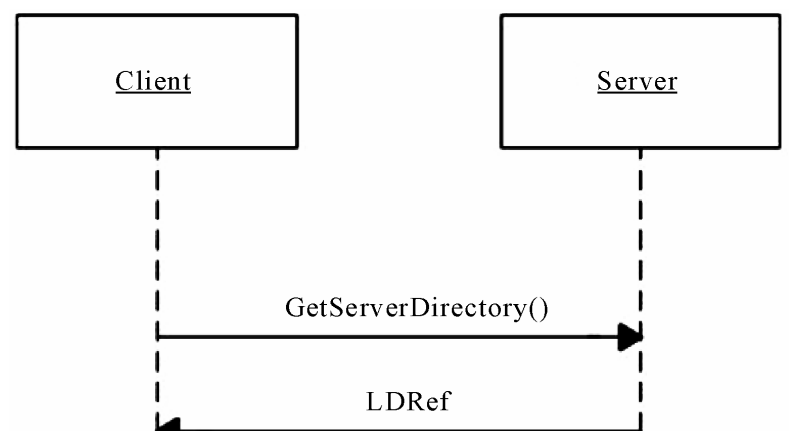

Figure 10. Method GetServerDirectory.

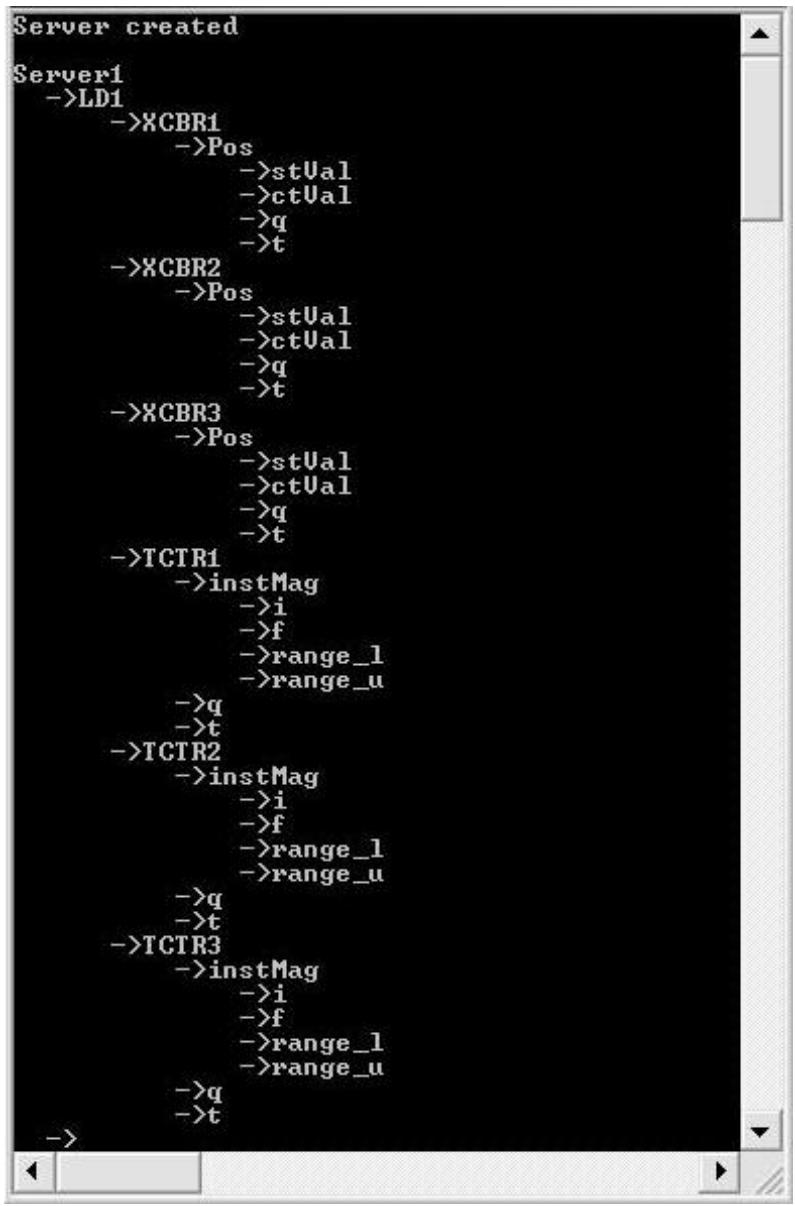

Figure 11. Server application at process level.

plans to utilize new communication technology following the IEC 61850 standard is implemented. It illustrates basic concepts of the norm and describes how these concepts can be used in the development and implementation of tools related to SAS.

Moreover, Standard IEC 61850 based IED modules were created and functionality improvements of SAS based on the standard were discussed.

The modules implemented over standard protocols illustrate that the standardization of data names, the crea- 


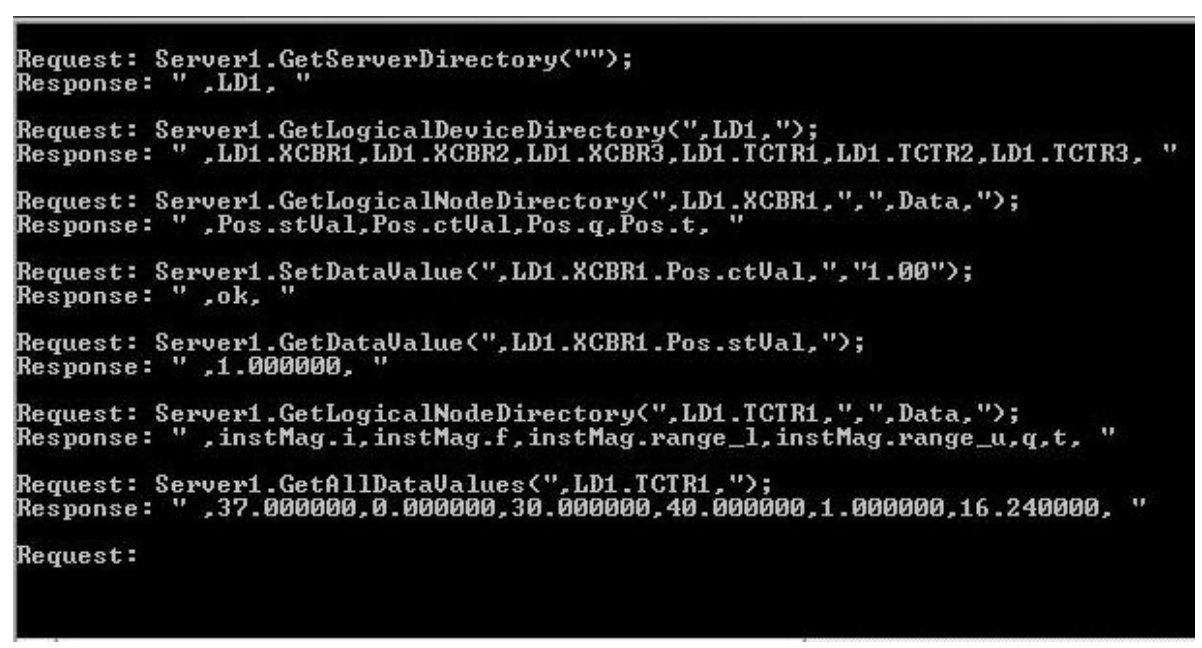

Figure 12. Client application at bay level.

tion of a comprehensive set of services, and the definition of a process bus offer benefits for the design of a substation automation system.

Finally, the implementation was developed based on a set of didactic laboratories providing undergraduate as well as graduate students the opportunity to carry out projects and laboratory work on communications in a real distribution system. The "real-world" nature of the implemented projects, and the exposition of student to a real substation, make a very valuable contribution to any power engineering curriculum

\section{References}

[1] V. Gungor and F. Lambert, "A Survey on Communication Networks for Electric System Automation," Computer Networks, Vol. 5, No. 1, 2006, pp. 877-897. doi:10.1016/j.comnet.2006.01.005

[2] M. Kezunovic, "Substation Automation Research Frontiers," Proceedings of the Power Systems Conference and Exposition, Seattle, 15-18 March 2009, pp. 1-2. doi:10.1109/PSCE.2009.4840104

[3] N. Nair and L. Zhang, "Smartgrid: Future Networks for New Zealand Power Systems Incorporation Distributed Generation,” Energy Policy, Vol. 37, No. 9, 2009, pp. 3418-3427. doi:10.1016/j.enpol.2009.03.025

[4] T. J. Hammons, "Integrating Renewable Energy Sources into European Grids," Electric Power and Energy Systems, Vol. 30, No. 8, 2008, pp. 462-475. doi:10.1016/j.ijepes.2008.04.010

[5] D. Dollen, "Report to NIST on the Smart Grid Interoperability Standards Roadmap,” Electric Power Research Institute, Palo Alto, June 2009.
[6] EPRI, “Guidelines for Implementing Substation Automation Using IEC61850, the International Power System Information Modeling Standard," Electric Power Research Institute, Palo Alto, 2004.

[7] R. Venkata, S. Pahwa and R. Christie, "What Future Distribution Engineers Need to Learn,” IEEE Transactions on Power Systems, Vol. 19, No. 1, 2004, pp. 17-23. doi:10.1109/TPWRS.2003.821017

[8] R. Kirkman, "Development in Substation Automation Systems," Proceedings of International Conference on Intelligent Systems Applications to Power Systems, Toki Messe, 5-8 November 2007, pp. 1-6. doi:10.1109/ISAP.2007.4441690

[9] T. Sidhu and Y. Yujie, "Modelling and Simulation for Performance Evaluation of IEC61850-Based Substation Communication Systems," IEEE Transactions on Power Delivery, Vol. 22, No. 3, 2007, pp. 1482-1489. doi:10.1109/TPWRD.2006.886788

[10] IEC-61850-6, “Communication Networks and Systems in Substations, Part 6: Configuration Description Language for Communication in Electrical Substations Related to IEDs,” International Electrotechnical Commission, Geneva, SW, 2004.

[11] R. E. Mackiewicz, “Overview of IEC61850 and Benefits,” 2006 IEEE Power Systems Conference and Exposition, Atlanta, 29 October-1 November 2006, pp. 623-630. doi:10.1109/PSCE.2006.296392

[12] IEC-61850-7, “Communication Networks and Systems in Substations, Part 7-420: Basic Communication Structure-Distributed Energy Resources Logical Nodes,” International Electrotechnical Commission, Geneva, 2009.

[13] KalkiManager. “Software SCL,” 2011. www.kalkitech.com 